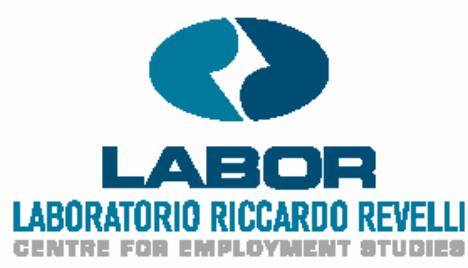

Working Paper no. 70

\title{
Should (and Could) We Ban Prescriptions?
}

\author{
Matteo Richiardi
}

November 26, 2007 


\title{
Should (and Could) We Ban Prescriptions?
}

\author{
Matteo Richiardi *
}

November 26, 2007

\begin{abstract}
In a simple model of social interaction I analyze the welfare effects of positive (prescriptive) and negative (proscriptive) social norms, together with the private incentives for their enactment. I find that imposing no law is socially optimal when individual actions have no significant externalities, while bans become socially optimal as the externalities increase. Prescriptions are generally the worst choice, but when the externalities involved are very high. However, in the political arena support is rarely won for non intervention or for a ban, since an alternative majority will generally be found in favor of some prescription. This remains true even when strategic voting is considered, and provides an argument for the idea that a liberal state cannot be liberally enforced by rational voters.
\end{abstract}

Keywords: Norms, Bans, Liberalism, Anarchy, Strategic voting.

\section{JEL Classification: D72.}

*Università Politecnica delle Marche and Collegio Carlo Alberto - LABORatorio R. Revelli. Email: m.g.richiardi@univpm.it. I wish to thank David Bertolini, Bruno Contini, Andrea Moro, Vernon Smith, Andrea Vindigni and Alberto Zazzaro for helpful comments and suggestions on earlier versions of this work. Responsibility for the content of this article remains exclusively my own. Financial support from the MIUR - PRIN 2005 program is gratefully acknowledged. 


\section{Introduction}

People stay together because they can benefit from mutual interaction. Of course, they can also suffer from these interactions. However, what is important for the analysis conducted below is that, living in societies, they can hardly escape to interact with each other. No matter how we behave, we affect other people's well being, and others affect us.

Given interaction, people have always tried to act in such a way to condition others' behavior, and make it less offensive, or more beneficial. Laws are explicitly aimed at this.

A problem then arises on how individual action should be regulated. Is it better to impose a behavior that is regarded as beneficial by most members of a community or ban a behavior that is considered harmful? When, on the other hand, should be preferred not to intervene at all, and let individuals behave as they like? These questions motivate the first part of the paper, where a normative analysis of three different forms of policies - no intervention, bans, and prescriptions - imposing different constraints on individual behavior is presented. The null option, which we might as well call natural order, or anarchy, means that no constraints at all are imposed on individual behavior. Bans refer to a prohibition to act in some specific way, which leaves individuals the choice between other alternatives. As such, they recall a liberal approach to regulation. Prescriptions are obligations to follow a specific behavior, and maintain a flavor of socialism.

The remaining of the paper confronts the answers to the questions above with what happens when people are allowed to vote. Are private incentives such that the social welfare is maximized? Or, conversely, are we doomed to implement laws that are even worse than the natural order?

I show that on average prescriptions are the optimal policy only when in- 
dividual actions have very strong (positive or negative) externalities. Bans are likely to be optimal when the externalities are medium to strong, while anarchy is better when externalities are low to medium. These statements are stochastic because which policy is actually the best, from an utilitarian perspective, depends on the distribution of individual preferences.

However, private incentives are such that a long-lasting consensus for nonintervention or for a ban is very rarely found, and prescriptions are agreed upon instead, even when this is not optimal.

The intuition is as follows. Limiting individual freedom comes always at a cost in terms of the utility of the specific individuals being constrained, and thus - ceteris paribus - also in terms of aggregate utility. If few people are harmed by a specific action, as is the case when the action produces a low level of externalities, the best policy is not to intervene. As the external effects increase, a ban on that action is generally to be preferred to an obligation to play some other action because it leaves more choices open, some of which might give a higher utility not only to those who are acting but also to other people in the society, with respect to the obligation to play one specific alternative. The optimality of prescriptions comes out of a specific assumption of the model, namely that the payoffs are symmetric: if one action produces a lot of harm to someone, there must be some other action that gives him/her a lot of good — stated differently, an individual cannot be affected by negative externalities only. In this case, if the level of externalities is high enough it becomes optimal to force individuals to play the action that is preferred by most of the people, irrespective of any other consideration. What is most interesting however is that if individuals are asked to vote among all different policy options and we assume that they vote sincerely, they would always support a prescription. This is true irrespective of the assumption of symmetric preferences, since they are always 
better off if their most preferred choice is established by law: put differently, everyone would prefer to be voted as a dictator.

As the relative minority realizes that a disliked prescription is going to be enacted, incentives for strategic voting arise. Some new majority might coordinate on voting a different prescription, or a ban. By considering a simple mechanism of probabilistic voting I show that non-intervention or limited intervention can be (strategically) supported only if the number of voters is limited, and only for some specific distribution of preferences in the society. As the size of the population increases, the effect of any individual vote becomes almost negligible and the ability for the relative minority to achieve coordination e.g. on a ban decreases: accordingly, the incentives for strategic voting become more limited, and the outcome converges to the non-strategic one: only prescriptions win support.

The paper is organized as follows: section 2 describes how this paper relates to the literature; section 3 describes the model; section 4 provides a normative analysis by comparing bans and prescriptions with respect to an utilitarian view of social welfare; section 5 analyzes what happens with binary competition, i.e. when competing policies are evaluated only pairwise; section 6 shows that when the different policies are jointly evaluated non-strategic voting always leads to the implementation of prescriptions, describes the mechanism of strategic voting considered and shows that even with strategic voting prescriptions remain the most likely outcome. Section 7 concludes.

\section{The literature}

This paper relates to two different strands of the literature: the one on the role of the law, liberalism and institutional design and the one on social choices and voting mechanisms, in particular with respect to the properties of majority 
ruling.

The Austrian and libertarian traditions have long stressed the virtues of minimal state intervention [von Hayek, 1960, Nozick, 1974]. However, their arguments rely mainly on a philosophical approach: liberty is good for its own sake, and individual freedom, but for a few limitations, is considered intangible. Freedom is a natural right, deriving ultimately either from some self-evident human trait or directly from God; it is never to be intended as a creation of human societies or a grant of governments. As such it cannot be traded against some other value and evaluated with respect to a somehow defined concept of social welfare.

Social contract theorists such as Hobbes, Locke or Rousseau all believed in a natural law and in natural rights. In this view the role of the state, to which specific monopoly powers are consensually transferred, is only to protect individuals from each other through the legitimate use of physical force.

This approach is at the heart of the English and American legal traditions, the principles of natural law being expressed, explicitly or implicitly, in such documents as the Magna Charta and the United States Declaration of Independence:

We hold these truths to be self-evident, that all men are created equal, that they are endowed by their Creator with certain unalienable Rights, that among these are Life, Liberty and the pursuit of Happiness. (The Unanimous Declaration of the Thirteen United States of America, 1776)

Followers of these traditions share a concept of negative liberty [Berlin, 1958], which refers to an individual's freedom from being subjected to the authority of others. In this negative sense one is considered to be free to the extent to which no person interferes with his or her activity: 
a free man is he that $[\ldots]$ is not hindered to do what he hath the

will to do. (Thomas Hobbes, Leviathan, ch. 21, 1651)

In the terms of the model proposed here, this view inclines either to supporting no government intervention at all, or negative obligations as expressed by bans on specific harmful actions.

On the contrary, supporters of positive liberty, interpreted as the freedom to achieve certain ends, might accept that the state has to force some behaviors upon people in order to make those ends at hands. Hence they would not object in principle to policies contemplating positive obligations, as the prescriptions considered in the model. ${ }^{1}$

Note that the positive and negative approach to social norms have a parallel with a positive and negative approach to moral norms, as exemplified by the two golden rules of Judaism ("don't do others what you don't like them do unto you") and Christianity ("do others what you would like them do unto you"). ${ }^{2}$

The present paper creates a bridge between this philosophical approach and the political economy literature, providing an utilitarian argument in favor of a more liberal state ${ }^{3}$. However, it also raises some doubts that such a liberal state might be agreed upon by means of a democratic voting process ${ }^{4}$, and indeed helps explaining why excessive regulation is often complained.

The paper is also related to the new institutional economics (NIE) literature [Williamson, 2000], which has specifically focused on the analytical investigation of different institutional frameworks. However, the main concern of NIE has been the definition and enforcement of property rights, mainly at what

\footnotetext{
${ }^{1}$ An echo of the distinction between a positive and a negative view of liberty can be found in the distinction between a process aspect and an opportunity aspect of liberty [Sen, 1999].

${ }^{2}$ The implications of these two norms for social welfare has been investigated in [Richiardi, 2006], in a model that is a particular case of the one presented here.

3 the utilitarian tradition in welfare economics, which dates back to the work of [Bentham, 1789], [Edgeworth, 1881], [Marshall, 1890] and [Pigou, 1920], allows for making judgments over social interest by comparing individual utilities

${ }^{4}$ which rules out the possibility of interpersonal comparisons: one head, one vote
} 
Williamson calls level 3, i.e. the level of governance.

The present paper adds to this literature by stressing the importance of analyzing different forms of legislative action. Specific institutional arrangements at level 2, the level of constitutions and general laws defining the rules of the game, might prove effective in binding further legislative choices thus allowing better types of laws to be implemented at lower tiers. ${ }^{5}$

From yet another perspective, and complementary to the philosophical literature stressing that individual freedom should not be traded in order to achieve some supposedly better social outcome, Sen's impossibility theorem [Sen, 1970] proves that it is not possible to have a social decision function that satisfies at the same time liberalism ${ }^{6}$ and Pareto optimality ${ }^{7}$. Rather then dispensing with the requirement of liberalism, Sen concluded that economists should not insist too much on Pareto efficiency, which however is the only welfare criterion remaining when interpersonal comparisons of utilities are ruled out.

Sen's approach is axiomatic. Instead, I look at the private incentives to vote, without imposing any requirement on the outcome. What I show is that, unless they are specifically safeguarded by some higher order law as a constitution, liberal values are at risk to be sacrificed to individual self-interest, empowered by the dictatorship of the majority. The democratic process produces a result that is Pareto optimal (any change in the law would harm someone) but is not liberal and, in many cases, not efficient from an utilitarian perspective.

Other unpleasant properties of majority ruling were already recognized in the 18th century by the Marquis of Condorcet [Condorcet, 1785], who stressed the problem of finding a stable outcome under the majority rule by showing how

\footnotetext{
${ }^{5}$ From a political economy perspective [Persson and Tabellini, 2003] investigate the empirical evidence of the economic effects of constitutions. Here I totally abstract from the economic incentives different policies might induce, and look only at individual preferences over those policies.

6 interpreted as allowing each individual to be decisive over at least some alternatives

${ }^{7}$ plus the conditions of unrestricted domain
} 
pairwise voting (binary agenda) over policy alternatives may fail to produce an overall winner. ${ }^{8}$

Ways out of the Condorcet paradox involve restricting preferences ${ }^{9}$, dispensing with the so-called open agenda assumption ${ }^{10}$ or dropping the assumption of pairwise comparison.

However, both agenda manipulation and non-pairwise policy evaluation provide strong incentives for strategic voting, i.e. non-sincere preference revelation. Indeed, [Gibbard, 1973] and [Satterthwaite, 1975] show that any democratic decision-making mechanism involving more than two options, including the majority rule, provides incentives for strategic voting. ${ }^{11}$

Strategic voting poses a problem because it might lead to different outcomes depending on how individual behavior is modeled, and in particular on the specific assumptions on how expectations are formed.

Strategic voting is indeed common in many real world situations. For instance, in first-past-the-post elections a left-wing voter may vote for a popular moderate candidate over an unpopular leftist candidate in order to help defeat a strong right-wing candidate ${ }^{12}$. In those proportional representation systems that include a minimum percentage of votes that a party must achieve to receive any seats, people might vote strategically for a minor party to prevent it from dropping below that percentage, which would make the votes it does receive use-

\footnotetext{
${ }^{8}$ The Condorcet paradox is an exemplification of the famous Arrow's impossibility theorem [Arrow, 1950], and more in general of the difficulties in formulating social preferences on a narrow informational base, which rules out interpersonal comparisons.

${ }^{9}$ see [Black, 1948], [Gans and Smart, 1996] and [Rothstein, 1990] for one-dimensional policies; [Grandmont, 1978], [Plott, 1967], [Davis et al., 1972] and [Hinich et al., 1972] for the multi-dimensional case

${ }^{10}$ for instance by imposing a limited number of pairwise votes

${ }^{11}$ The two theorems state that any voting method which is completely strategy-free must be either dictatorial or non-deterministic. Non-determinism is exemplified by the random ballot voting method, which assigns the outcome after looking at a single vote randomly selected. The random ballot method may of course result in different choices being selected if applied multiple times to the same set of ballots.

12 this is the basis for the Duverger's law [Duverger, 1972], suggesting that first-past-the-post election systems generally leads to two-party systems
} 
less for the larger political camp that party belongs to. Finally, strategic voting is even more an issue when only a few players are involved, as in corporate boards, ministry councils, etc.

A widely used approach to deal with strategic voting in the political economy literature is to consider probabilistic voting [Hinich, 1977], [Coughlin and Nitzan, 1981], [Ledyard, 1981], [Ledyard, 1984]. Probabilistic models assume that individuals assign probabilities to the voting behavior of other people.

In this paper I adopt a similar approach. However, probabilistic voting models generally consider uncertainty on the part of the candidates (who have to choose their electoral platform), for instance because some individuals might vote sincerely due to the force of ideology, or some others might not even dare to turn out to the ballot. In the model I propose here there are no candidates or, put differently, the electoral platforms are exogenously given and only voters' behavior is modeled. As is usual in models of voting behavior, I make the assumption of common knowledge of the electoral situation. ${ }^{13}$ However, in my model voters learn about the popularity of the competing policy alternatives, through repeated interactions. Individuals weight the payoffs they get under each policy by the probability of that policy being enacted, and they estimate this probability adaptively by looking at the share of the vote the same policy has attracted in the last competition. They finally vote for the option to which they attach the highest expected payoff. The implications of such a mechanism, which similarly to [Myatt, 2007] is able to account for the fact that the trailing challenger suffers only incomplete strategic desertion, are investigated by means of an agent-based simulation model [Tesfatsion, 2006]. ${ }^{14}$

The assumption of repeated interaction is of course inadequate for most

\footnotetext{
${ }^{13} \mathrm{~A}$ model which assumes that voters can only observe a private signal about the popularity of competing candidates is [Myatt, 2007].

${ }^{14}$ From a methodological point of view, the application of computer simulations to the analysis of voting outcomes with strategic behavior was pioneered by [Tullock and Campbell, 1970], who looked at cycles in small committees.
} 
real situations ${ }^{15}$. However, it makes sense here because I'm only interested in the equilibrium outcome. Out-of-equilibrium electoral rounds should then be thought of as an "as if" modeling device to account for individual learning. Moreover, it should be remembered that I do not intend to present here a complete new theory of strategic voting, but rather a simple mechanism to test if the implications of my model are robust to strategic behavior.

\section{The model}

With the aim to keep the model minimal, I consider only three alternatives, over which individuals have different preferences. Restricting our attention to three options at a time is a reasonable simplification, which - as it will be shown below - preserves most of the interesting dynamics inherent in the social choice.

In a model with just two alternatives there would be no difference between banning one option and imposing the other. On the other hand, in a model with more than three options additional policies become possible, like banning more than one alternative or restricting individual choice among a small set of alternatives. These policies however look like a half-way between a pure negative (forbidding one and only one action) and a pure positive (imposing one and only one action) obligation. It can therefore be expected that their properties would be a mixture between the properties of pure negative and pure positive obligations analyzed below.

The three actions are labeled A, B and C. The distribution $P$ of individual preferences over these actions can be summarized by the 6 -tuple $\left\{p_{1}, \cdots, p_{6}\right\}$, each number representing the fraction of the population sharing the same combination of the most preferred and the least preferred action (table 1).

\footnotetext{
15 although it can be argued that it might work for countries like e.g. Italy, with 18 general elections, including those for the European Parliament but excluding referendums, between 1946 and 2006, or Israel, with 17 general elections between 1949 and 2006, or Switzerland,
} 


\begin{tabular}{cccc}
\hline Type & Most preferred & Least preferred & Share \\
\hline 1 & $\mathrm{~A}$ & $\mathrm{~B}$ & $p_{1}$ \\
2 & $\mathrm{~A}$ & $\mathrm{C}$ & $p_{2}$ \\
3 & $\mathrm{~B}$ & $\mathrm{~A}$ & $p_{3}$ \\
4 & $\mathrm{~B}$ & $\mathrm{C}$ & $p_{4}$ \\
5 & $\mathrm{C}$ & $\mathrm{A}$ & $p_{5}$ \\
6 & $\mathrm{C}$ & $\mathrm{B}$ & $p_{6}$ \\
\hline
\end{tabular}

Table 1: Distribution of preferences

Individual utility is affected by own actions and by the actions played by whom the individual is interacting with. Own actions affect $n \in[0, N]$ other people, randomly drawn from a population of size $N$.

Both individual utility and aggregate welfare are defined only in terms of the expected (individual or total) payoff from one random interaction, $\pi$.

Individuals get a payoff of $\alpha$ when they play what they like and a payoff of $-\alpha$ when they are forced to play what they don't like. They get 0 when they play their second choice. Moreover, they cause a payoff of $\beta$ to each passive opponent should $\mathrm{s} /$ he approve what they have done, and a payoff of $-\beta$ should $\mathrm{s} /$ he disapprove. They cause a payoff of 0 if the opponent ranks the action as his/her second choice.

Individual preferences are private information, and communication before interaction is not possible. This excludes the possibility of contracting out $\grave{a} l a$ Coase [Coase, 1960] the externality problem.

There is no political intermediation. Individuals can ask for a vote on any combination of competing policy options, which include both prescriptions and proscriptions. If no majority is reached, no obligation is passed. People are allowed to vote strategically, i.e. they do take into consideration that their best option might be out of reach and give support to some least preferred policy. Incentives to break the law are not considered (suppose the cost of punishment where on average a vote is called for (on specific issues) 4 times a year 
is high enough).

\subsection{Discussion}

The structure of the payoffs considered can be better evaluated after discussion of two simplifying assumptions concerning (i) symmetry, and (ii) homogeneity.

The symmetry of the payoffs around 0 poses no problems when looking at which action is played, since only the ranking is relevant in this case. It does however affect which policy is voted, and which policy ranking comes out of social welfare considerations. It would be however easy to accommodate for asymmetric evaluations by considering the following payoff matrix:

\begin{tabular}{ccc}
\hline $\begin{array}{c}\text { Action } \\
\text { rank }\end{array}$ & $\begin{array}{c}\text { Internal } \\
\text { payoff }\end{array}$ & $\begin{array}{c}\text { External } \\
\text { payoff }\end{array}$ \\
\hline 1 & $\alpha_{1}$ & $\beta_{1}$ \\
2 & $\alpha_{2}$ & $\beta_{2}$ \\
3 & $\alpha_{3}$ & $\beta_{3}$ \\
\hline
\end{tabular}

Table 2: General payoff matrix. Internal payoff refers to the utility individual $m$ gets when s/he plays action $a$. External payoff refers to the externality individual $m$ receives when another individual plays action $a$.

This would also allow to explicitly model a taste for diversity, i.e. the case when agents' most preferred actions do not coincide with what they would wish others to do (the rank in terms of the $\alpha$ s might differ from that of the $\beta \mathrm{s}$ ).

For the sake of simplification I make the assumptions (1) $\beta_{a}=k \alpha_{a}$ for all actions $a$, and (2) $\alpha_{3}=-\alpha_{1}$, but it would be easy to accommodate for the general structure of table 2 . Note that assumption (1) does not necessary imply a preference for conformism, as actions can be appropriately redefined in order to fit in this simplified framework. This will be clarified below when discussing possible applications of the model. The additional normalization $\alpha_{2}=0$ is innocuous.

As for what concerns point (ii), the assumption that $\alpha$ and $\beta$ are homoge- 
neous in the population can also be easily relaxed, provided that we are willing to assume that the individual $\alpha_{m}$ and $\beta_{m}$ are uncorrelated with the individual types. Considering heterogeneity in $\alpha$ and $\beta$ in this case only implies to replace $\alpha$ and $\beta$ with their average value $\bar{\alpha}$ and $\bar{\beta}$ in eqs. $1-3$ below, and $\beta$ alone with its average value $\bar{\beta}$ in eqs. $6-12$.

Moreover, under the plausible assumption that $\beta_{m}=k \alpha_{m}$ (whoever has a bigger $\alpha$ has also a bigger $\beta$, and vice versa) heterogeneity changes neither actions nor votes, since they are not based on interpersonal comparisons.

As a an example of a practical application of the model, consider the case of rape-induced pregnancies. Suppose the alternatives are (i) abort, (ii) leave the child for adoption and (iii) keep the child. Any decision is likely to affect not only the utility of those who take it, but also other people (who might disregard it on moral grounds, or like it as a signal that the option is truly available), and which in turn might try to exert an influence on the choice. Interaction here is in principle global, the notice of the action being enough to cause a reaction.

A second example is city color plans or, more in general, zoning laws. Specific restrictions on house colors or development types (residential, business or commercial buildings, parks, etc.) are often imposed in many urban areas. These restrictions might obviously involve either prescriptions or proscriptions. This example is also well suited to discuss how a taste for diversity could be accounted for while keeping the simplifying assumption $\beta_{a}=k \alpha_{a}$ : simply define one of the available options as "Paint your house a different color from your neighbor's".

One more example is dressing code. France for instance has passed in 2004 a law forbidding people holding public offices and scholars to show off religious signs (such as the Muslim veil, the Jewish kippah, etc.). The ban, which was promoted to foster integration and fight integralism, has prompted a wide debate 
and a fierce opposition, inside and outside France, exactly on grounds of being illiberal. However, stronger prescriptions, like wearing school uniforms, are usual in many countries (as in British schools).

Finally, note that $\alpha=0{ }^{16}$ implies individuals either don't have or cannot predict any consequence from their actions to their own utility. As an example, consider the case of you getting to know that your best friend's girlfriend has a relationship with some other guy. What should you do? You can either tell your friend, or speak with his girlfriend, perhaps speak with the other guy, or say nothing (which is by the way different from not interacting at all). In either case the well being of your friend is going to be affected $(\beta>0)$. What's most, you will probably not know how your friend would like you to behave: asking him being equivalent to telling him the truth.

\section{Social welfare}

Purely altruistic individuals with $\alpha=0$ would choose their action by considering some prior about the distribution of types $P$. On the other hand, selfish individuals with $\alpha>0$ would always play their preferred action, if possible. If this is not allowed, they would choose their second best option, and only if forced to they would play their least preferred action.

The individual expected payoffs under different policies are reported in the Appendix. The corresponding expressions for the social welfare are simply obtained by multiplying the expected payoffs for each individual type by the relative frequency of that type, and then summing up.

Under the Null policy, the expected social welfare associated to the action of one random individual in the population is:

\footnotetext{
${ }^{16}$ as in [Richiardi, 2006]
} 


$$
\begin{aligned}
\pi_{0}= & \alpha+\beta n[ \\
& \left(p_{1}+p_{2}\right)\left(p_{1}+p_{2}-p_{3}-p_{5}\right) \\
+ & \left(p_{3}+p_{4}\right)\left(p_{3}+p_{4}-p_{1}-p_{6}\right) \\
+ & \left.\left(p_{5}+p_{6}\right)\left(p_{5}+p_{6}-p_{2}-p_{4}\right)\right]
\end{aligned}
$$

To see why, consider the case of a type 1 or type 2 individual (they both love A). Without restrictions they will always play $\mathrm{A}$, and get $\alpha$ for themselves. In addition, they will cause a payoff of $\beta$ in $\left(p_{1}+p_{2}\right) n$ individuals, and a payoff of $-\beta$ in $\left(p_{3}+p_{5}\right) n$ individuals. Note that the probability of choosing randomly a type 1 or type 2 individual is $\left(p_{1}+p_{2}\right)$. The expected outcomes for the case when $\mathrm{B}$ or $\mathrm{C}$ are played account for the remaining terms.

Second, let's suppose a prescription is imposed. To fix ideas, consider the case when $\mathrm{A}$ is law. The expected payoff for an active player will be $\pi_{\text {A,active }}=$ $\left(p_{1}+p_{2}-p_{3}-p_{5}\right) \alpha$, while $\mathrm{s} /$ he will cause an expected payoff $\pi_{A, \text { passive }}=$ $\left(p_{1}+p_{2}-p_{3}-p_{5}\right) \beta$ to any of the $n$ passive players $\mathrm{s} /$ he is interacting with. Hence:

$$
\pi_{A}=(\alpha+\beta n)\left(p_{1}+p_{2}-p_{3}-p_{5}\right)
$$

The expressions for the case when B or C becomes compulsory easily follow.

Third, let's consider the case of a ban. To fix ideas, focus on the case when $\mathrm{C}$ is forbidden. The corresponding expression for social welfare is:

$$
\begin{aligned}
\pi_{\bar{C}} & =\alpha\left(p_{1}+p_{2}+p_{3}+p_{4}\right) \\
& +\beta n\left(p_{1}+p_{2}+p_{6}\right)\left(p_{1}+p_{2}-p_{3}-p_{5}\right) \\
& +\beta n\left(p_{3}+p_{4}+p_{5}\right)\left(p_{3}+p_{4}-p_{1}-p_{6}\right)
\end{aligned}
$$

All individuals but those who like $\mathrm{C}$ are able to do what they prefer. This accounts for the first term. Those who play A (type 1 and 2 plus type 6 , who 


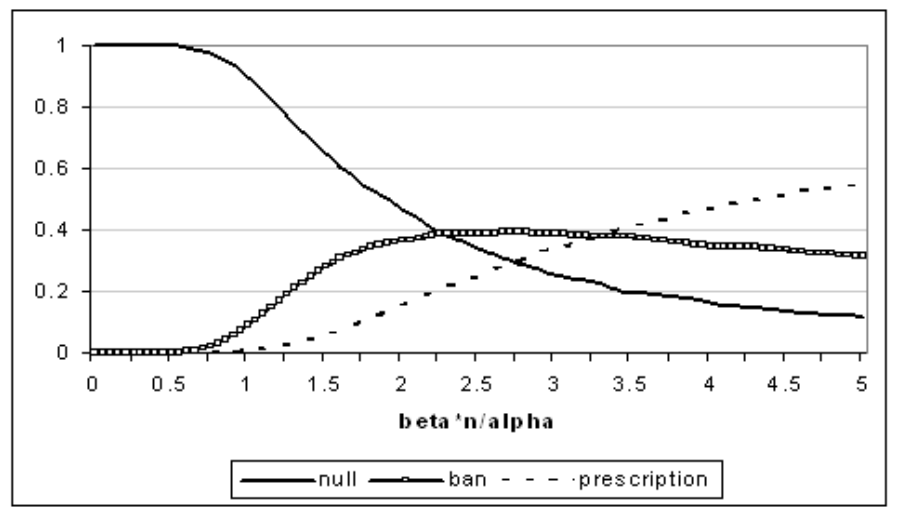

Figure 1: Best policies. Each point is the probability, for an unknown underlying preference configuration, that a specific policy is socially optimal. The probabilities may sum to less than 1 because for specific preference configurations different policy types may lead to the same level of social welfare. No winner is assigned in this case.

have to play their second choice) cause positive externalities in type 1 and type 2 individuals, and negative externalities in type 3 and type 5 individuals (second term). Those who play B (type 3 and 4 plus type 5 , who have to play their second choice) cause positive externalities in type 3 and type 4 individuals, and negative externalities in type 1 and type 6 individuals (third term). The expressions for the case when A or B become outlaw involve but straightforward modifications.

Comparing 6 different policies (3 prescriptions, 3 bans) plus the Null for any possible distribution of individual preferences and any combination of $\alpha$, $\beta$ and $n$ proves a difficult task analytically. Instead, I have normalized $\alpha$ to 1 and simulated the outcomes by letting each $p_{t}, t=1, \ldots, 6$ and $\beta n$ change by increasing steps of .05. This leads to the evaluation of 5,440,115 different cases. The results are summarized in figure 1 .

Supposing no priors about the distribution of preferences in the population, from a social point of view it is better not to impose any restriction whatsoever 
on individual behavior if the overall level of externalities (the externality produced by an action, multiplied by the number of people affected) are not too high $(\beta n<2.25 \alpha)$. Bans are the most likely optimal policy if the overall level of externalities is between 2.25 and 3.25 times the level of internal (self-generated) utility, and prescriptions are the most likely optimal policy if the overall level of externalities is above 3.25 times the level of internal utility.

\subsection{Discussion}

The analysis of the previous paragraph suggests to look at a measure of total externalities, $\beta n$. Other things being equal, increasing the number of persons affected by the action would thus increase the likelihood that it is socially optimal to intervene.

In the abortion example passive players might well be very numerous; however, $\beta$ is likely to be very small with respect to $\alpha$. In the case of zoning laws $\alpha$ is probably smaller but the number of people $n$ affected by the action is also smaller.

Prescriptions are optimal only when a large number of people love a given action, but still there is a minority that would otherwise choose some other action, and the (negative) externalities from this minority are relevant. For instance, if $\beta n=\alpha=1$ and the type distribution is $p_{1}=.55 ; p_{2}=.25 ; p_{3}=$ $0 ; p_{4}=.1 ; p_{5}=0 ; p_{6}=.1$ prescribing A becomes optimal: $80 \%$ of the population loves A, however the remaining $20 \%$ of people would choose actions that the majority dislikes. However, in this case the gain over the Null policy is quite limited $(+2.56 \%)$, and the gain over imposing a ban on B (which is disliked by $65 \%$ of the population) even more so (only $+0.31 \%$ ).

To see this in more general terms, it is convenient to represent the distribution of preferences in the society as a single point in a three dimensional space, 
where the axes are labeled $a, b$ and $c$. The $a$ coordinate is found by counting all individuals who love $\mathrm{A}$, and subtracting all individuals who hate $\mathrm{A}$. The result is then normalized to the size of the population. Similarly for the other two coordinates. ${ }^{17}$ Hence,

$$
\begin{aligned}
& a=p_{1}+p_{2}-p_{3}-p_{5} \\
& b=p_{3}+p_{4}-p_{1}-p_{6} \\
& c=p_{5}+p_{6}-p_{2}-p_{4}
\end{aligned}
$$

and $a+b+c=0$.

Note that different distributions of preferences can lead to the same point in the space. For instance, the point in the origin is given not only by $p_{1}=$ $p_{2}=p_{3}=p_{4}=p_{5}=p_{6}=\frac{1}{6}$, but by any combination of preferences such that $p_{1}=p_{3} ; p_{2}=p_{5} ; p_{4}=p_{6}$. We can now define the extent to which preferences are homogeneous as the distance from the center of the sphere:

$$
d(a, b, c) \equiv d\left(p_{1}, \cdots, p_{6}\right)=\sqrt{a^{2}+b^{2}+c^{2}}
$$

Note that $d \in[0, \sqrt{2}]$ : all points lie inside a sphere around the origin. In the center of the sphere preferences are more fragmented; on the surface they are more unanimous.

Figure 2 shows that (i) for a given level of the externalities, the higher the level of homogeneity $d$ in the population the higher is the likelihood that it is optimal to intervene with a more coercive policy (first bans, then prescriptions), and (ii) the higher the level of externalities the lower is the level of homogeneity when it is likely to become optimal to intervene.

\footnotetext{
${ }^{17}$ This mechanism is equivalent to defining a Borda count social decision function.
} 

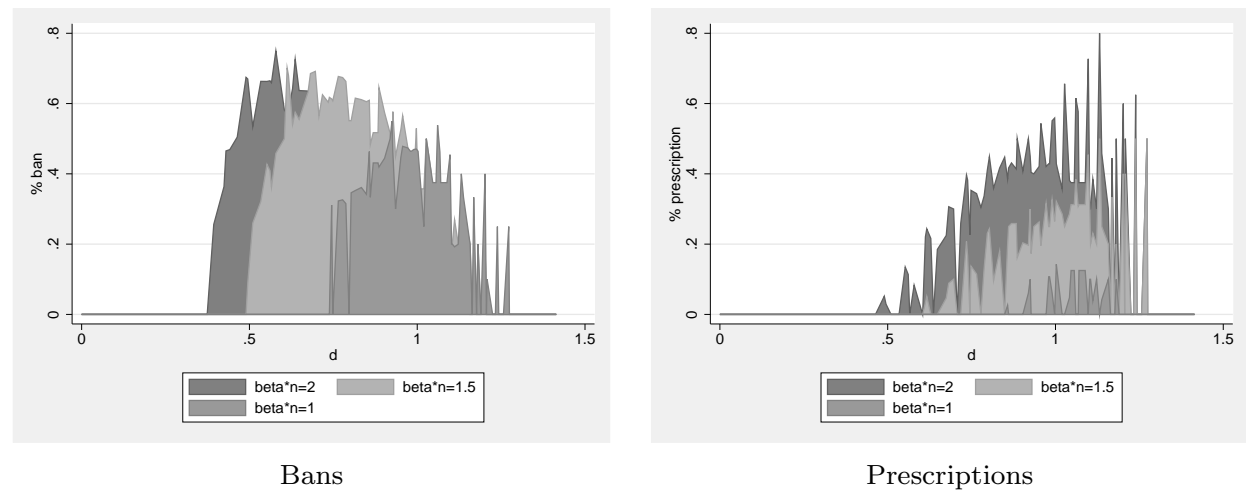

Figure 2: Probability, averaged over preference configurations that lead to the same distance measure $d$, that a ban (left panel) or a prescription (right panel) is optimal, for three different values of $\beta n$. The spikes are due to the fact that, given the specific grid chosen for exploring the space of possible preference distributions, some values of $d$ can be produced by more combinations, while others by less combinations, and some others cannot be produced at all. By decreasing the value of the step smoother figures are obtained.

\section{$5 \quad$ Binary agenda}

When comparing only two policy options strategic voting is not an issue, and rational individuals will always support the alternative that brings them higher utility, as reported in the Appendix. However, due to the multi-dimensional character of the model pairwise comparison of policy alternatives (binary agenda) leads to policy cycles: for any given policy, an alternative can generally be found that will get the majority support. Thus, a majority will ask for a referendum on the alternative, and will win it.

A typical policy transition matrix is reported in table 3 , where each cell $(i, j)$ contains the share of the voters supporting policy $j$ against policy $i$. A switch to the new policy takes place whenever the value in the corresponding cell is above .5 (bold values).

In the example, A will never be implemented; the Null and No A policies are on the other hand quite likely, since they can be defeated only by No A and 


\begin{tabular}{lccccccc}
\hline & \multicolumn{7}{c}{$t+1$} \\
$t$ & Null & No A & No B & No C & A & B & C \\
\hline Null & &. $\mathbf{5 6}$ & .47 & .44 & .32 & .41 & .27 \\
No A & .44 & & .47 & .44 & .32 & $\mathbf{. 5 3}$ & .47 \\
No B & $\mathbf{. 5 3}$ & $\mathbf{. 5 3}$ & & $\mathbf{. 5 3}$ & .44 & .41 & $\mathbf{. 5 6}$ \\
No C & $\mathbf{. 5 6}$ & $\mathbf{. 5 6}$ & .47 & & .47 & $\mathbf{. 5 3}$ & .27 \\
A & $\mathbf{. 6 8}$ & $\mathbf{. 6 8}$ & $\mathbf{. 5 6}$ & $\mathbf{. 5 3}$ & & $\mathbf{. 5 3}$ & $\mathbf{. 5 6}$ \\
B & $\mathbf{. 5 9}$ & .47 & $\mathbf{. 5 9}$ & .47 & .47 & & .47 \\
C & $\mathbf{. 7 3}$ & $\mathbf{. 5 3}$ & .44 & $\mathbf{. 7 3}$ & .44 & $\mathbf{. 5 3}$ & \\
\hline
\end{tabular}

Table 3: Policy transition matrix: each cell $(i, j)$ reports the share of the voters supporting policy $j$ against policy $i$. Bold values indicate that the transition takes place. Preferences are $p_{1}=.20 ; p_{2}=.12 ; p_{3}=.29 ; p_{4}=.12 ; p_{5}=.12 ; p_{6}=$ .15. Payoffs are $\alpha=\beta n=1$.

$\mathrm{B}$, respectively.

Note that if policy $i$ is defeated against policy $j$ it cannot be the case that in a subsequent vote the outcome is reversed: the sum of any two symmetric cells must be 1 .

\subsection{Replacement of bans with obligations}

It is interesting to note that a majority can always be found to replace a specific ban with an appropriate prescription, but the reverse is not necessarily true.

To see this suppose without loss of generality a ban on playing $\mathrm{C}$ is in place. Straightforward comparison of the individual payoffs under the different policies reported in the Appendix shows that types 1,2 and 6 will be in favor of replacing it with an obligation to play A, while types 3,4 and 5 would oppose the change. Thus, if $p_{1}+p_{2}+p_{6}>p_{3}+p_{4}+p_{5}$ the ban is replaced with an obligation to play $\mathrm{A}$. However, if this is not the case and $p_{1}+p_{2}+p_{6}<p_{3}+p_{4}+p_{5}$ a new absolute majority is found to replace the ban with an obligation to play B. Note that this is true irrespective of the values of $\alpha, \beta$ and $n .^{18}$ Hence, in pairwise comparisons an appropriate prescription can always be found to replace

\footnotetext{
${ }^{18}$ Of course for some preference distributions a prescription to play $\mathrm{C}$ might also be preferred to the ban.
} 
a specific ban.

Now, suppose again without loss of generality that a prescription to play $\mathrm{A}$ is in place. By comparing the different individual incentives reported in the Appendix we observe that:

- support will be found to switch to No B if $p_{3}+p_{5}+p_{6}>.5$;

- support will be found to switch to No C if $p_{3}+p_{4}+p_{5}>.5$;

- when A is confronted with No A, type 1 and 2 individuals will oppose the change, while type 3 and 5 will support it; type 4 will be in favor only if $\alpha+\beta n\left(-p_{1}+p_{2}+p_{3}+p_{4}-p_{5}-p_{6}\right)>0$, and type 6 will be in favor only if $\alpha+\beta n\left(p_{1}-p_{2}-p_{3}-p_{4}+p_{5}+p_{6}\right)>0$.

Numerical evaluation of all possible preference combinations obtained by letting again each $p_{t}$ change by increasing steps of .05 in $[0,1]$, and $\beta n$ change by increasing steps of .05 in $[0,2]$ shows a change to a ban will happen only in $64.47 \%$ of the cases. In other words, in pairwise comparisons it is not always true that an appropriate ban can be found to replace a specific prescription.

\section{Full Agenda}

Let's now turn to the case when all policy options are jointly evaluated (full agenda). Individuals are asked to vote for the policy they prefer: if no majority for any ban or prescription is found, the Null policy remains in place. It is trivial to see that the best (non-strategic) choice is to support an obligation prescribing to do one's own most preferred action. Thus, if $\max \left(p_{1}+p_{2}, p_{3}+p_{4}, p_{5}+p_{6}\right)<.5$ no policy is implemented; alternatively, a prescription is put in place (see table $4)$.

As already discussed, enlarging the set of possible alternatives provides strong incentives for strategic voting. In the framework of the present model, 


\begin{tabular}{cc}
\hline Policy & Supported by \\
\hline $\mathrm{A}$ & $p_{1}+p_{2}$ \\
$\mathrm{~B}$ & $p_{3}+p_{4}$ \\
$\mathrm{C}$ & $p_{5}+p_{6}$ \\
\hline
\end{tabular}

Table 4: Support for prescriptions, full agenda

a non trivial question is whether such a behavior leads to an increased support for bans or the Null policy.

To investigate this issue I adopt the following mechanism of strategic voting: individuals vote for the option that brings them higher expected payoffs; they compute these expected payoffs by discounting the payoffs they get under each policy by the probability of that policy being enacted; they hold adaptive expectations on this probability based on the results of the last vote. In addition, to allow for more strategy exploration each voter is assumed to vote randomly with probability $r .{ }^{19}$

This simple mechanism has some (emergent) pleasant properties. First of all, the number of non-sincere voters decreases with the size of the population, the outcome correspondingly approaches the result under non-strategic voting. Moreover, as the size of the population increases strategic voting is increasingly targeted at supporting the second most popular policy option. That is, as individuals realize it is more and more difficult to affect the outcome of the vote, some stop playing strategically and turn back to sincere preference revelation, while some others join their efforts and focus their support on the most likely alternative.

The implications of this voting strategy are analyzed by means of an agentbased simulation. ${ }^{20}$

\footnotetext{
${ }^{19}$ Varying this parameter however only affects the speed of convergence and the stability of the stationary state, a higher speed (a higher value of $r$ ) leading to a more perturbed outcome.

${ }^{20}$ The simulation is written in Java and makes use of JAS simulation platform [Sonnessa, 2004]. The code can be downloaded from http://utenti.dea.univpm.it/richiardi/code/Bans.rar or requested to the author.
} 


\begin{tabular}{|c|c|c|c|c|}
\hline Who & $\begin{array}{c}\text { When } \\
\text { t(0) } \\
\end{array}$ & What & & \\
\hline model & $\phi$ & initialize 0 & \multicolumn{2}{|c|}{ create $\mathrm{N}$ agents of random types } \\
\hline agents & $\phi$ & vote0: & $\begin{array}{l}\text { with prob. } r \\
\text { with prob. }(1-r)\end{array}$ & $\begin{array}{l}\text { wote randomly } \\
\text { for each policy option: } \\
\text { get } s=\text { share of votes for that policy in previous election } \\
\text { get } P=\text { expected payoffs if that policy is implemented } \\
\text { compute ex-ante expected payoffs = } s^{*} P \\
\text { vote for the policy with highest ex-ante expected payoffs } \\
\text { Tiebreak rule: if indifferent prefer null to bans to prescriptions }\end{array}$ \\
\hline model & $\phi$ & scrutiny0 & $\begin{array}{l}\text { implement win } \\
\text { record vote sha }\end{array}$ & \\
\hline
\end{tabular}

Figure 3: Simulation schedule

The schedule of the simulation is reported in figure 3. The number of agents, $N$, is set to 100 . The experimentation probability $r$ is set to .1 .

The first thing to note is that this voting strategy does not lead to policy cycles. Some distribution of preferences may lead to very close races, and due to the fraction of random voters policy switches are indeed observed; however, as soon as randomness is removed stability is obtained. Figure 4 exemplifies this.

As figure 4 shows, convergence, i.e. stationarity of the vote shares is immediately achieved if the population size is bigger than a handful of agents. Even with $N<10$ players convergence generally takes place within 300 periods. To allow for such situations in the analysis that follows each simulation run lasts for 500 periods (500 election rounds), and only the outcome of the last election is recorded. 32,000 simulation runs are performed, for different values of the population size $N \in\{5,10, \cdots, 40,100\}$. In each run preferences (i.e. types) are assigned randomly, while $\beta n$ is randomly extracted from $\{.2, .4, .6, .8,1\}$. Hence, according to the results of section 4 we are in a region where the socially optimal policy is anarchy. For the sake of brevity only outcomes for values of 


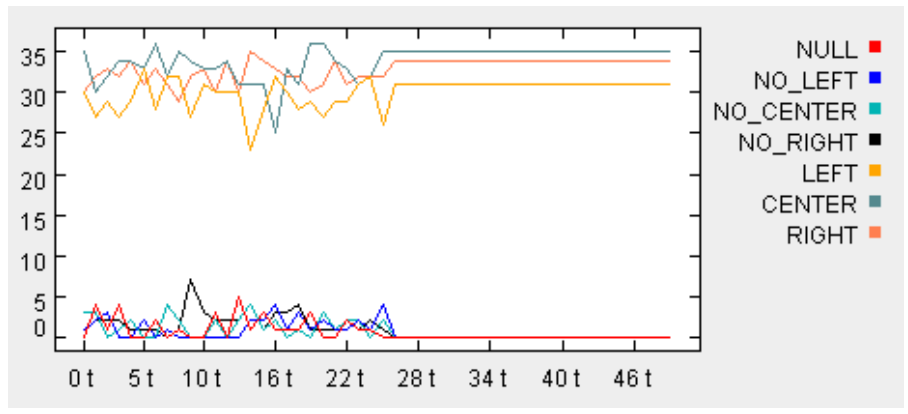

Figure 4: Probabilistic voting. One single simulation run consisting of 50 periods. Population size is 100 , with the following preference distribution: $p_{1}=.20 ; p_{2}=.11 ; p_{3}=.18 ; p_{4}=.17 ; p_{5}=.34 ; p_{6}=0 . \quad \alpha=1 ; \beta n=1$. $r=.1$ in the first 25 periods; $r=0$ in the last 25 periods.

$\beta n \in\{.2,1\}$ are reported.

Table 5 shows that the number of strategic voters in the population decreases as the population size increases, to reach a floor at about $45 \%$, irrespective of the values of $\beta$ and $n$. However, the share of the population that votes strategically in favor of a minority policy option increases. This is also shown in table 6 , where the fraction of strategic voters supporting each policy (ranked from the most to the least voted) is reported. As $N$ increases and the winning policy becomes less and less influenced by strategic voting, those individuals who still find attractive to play strategically do it in order to affect the choice of the most relevant alternative.

Note that these two characteristics of the specific probabilistic voting mechanism used in this paper are emergent phenomena, and could not easily be predicted knowing only the individual voting rule.

Finally, table 7 reports the outcomes of the electoral contest. Anarchy and bans have no chance of being implemented as soon as the population size grows over 30 individuals, and prescriptions are always voted. As expected, higher values of the externality parameter $\beta$ and of the number of affected people $n$ 


\begin{tabular}{|c|c|c|c|c|}
\hline \multirow[b]{2}{*}{$N$} & \multirow{2}{*}{$\begin{array}{c}\text { Strategic } \\
\text { voters }\end{array}$} & \multicolumn{2}{|c|}{ Voting with: } & \multirow[b]{2}{*}{ Obs. } \\
\hline & & majority & minority & \\
\hline \multicolumn{5}{|c|}{$\beta n=.2$} \\
\hline 5 & .74 & .63 & .11 & 832 \\
\hline 10 & .74 & .61 & .13 & 619 \\
\hline 15 & .63 & .46 & .18 & 774 \\
\hline 20 & .55 & .31 & .23 & 570 \\
\hline 25 & .49 & .25 & .24 & 815 \\
\hline 30 & .47 & .22 & .25 & 753 \\
\hline 35 & .46 & .21 & .24 & 793 \\
\hline 40 & .45 & .21 & .25 & 790 \\
\hline 100 & .46 & .21 & .25 & 501 \\
\hline \multicolumn{5}{|c|}{$\beta n=1$} \\
\hline 5 & .64 & .50 & .14 & 742 \\
\hline 10 & .62 & .44 & .18 & 622 \\
\hline 15 & .50 & .27 & .23 & 795 \\
\hline 20 & .47 & .22 & .24 & 620 \\
\hline 25 & .45 & .21 & .24 & 787 \\
\hline 30 & .44 & .21 & .24 & 768 \\
\hline 35 & .45 & .21 & .24 & 840 \\
\hline 40 & .45 & .21 & .24 & 784 \\
\hline 100 & .44 & .20 & .25 & 512 \\
\hline
\end{tabular}

Table 5: Fraction of strategic voters in the population

lead to a higher frequency of implementation of prescriptions even when the population size is (very) small.

\subsection{Discussion}

I have shown that when all policy options are jointly considered non-strategic behavior always leads to some prescription being selected. Strategic behavior could in principle change this outcome, but I have shown that in reality, except for decisions involving only a small number of agents, this never happens.

Of course, bargaining might totally internalize the externality problem and allow a recovery of the socially efficient outcome. However, the possibility to agree on side payments on voting decisions are limited, if not explicitly prohibited by the law. 


\begin{tabular}{|c|c|c|c|c|c|}
\hline \multicolumn{6}{|c|}{$\begin{array}{l}\text { Fraction of strategic voters } \\
\text { supporting policy ranked: }\end{array}$} \\
\hline$N$ & 1 & 2 & 3 & 4 & Obs \\
\hline \multicolumn{6}{|c|}{$\beta n=.2$} \\
\hline 5 & .73 & .14 & .06 & .00 & 832 \\
\hline 10 & .72 & .19 & .07 & .01 & 619 \\
\hline 15 & .60 & .23 & .10 & .05 & 774 \\
\hline 20 & .48 & .27 & .16 & .06 & 570 \\
\hline 25 & .44 & .29 & .17 & .06 & 815 \\
\hline 30 & .42 & .29 & .18 & .06 & 753 \\
\hline 35 & .41 & .29 & .18 & .07 & 793 \\
\hline 40 & .40 & .30 & .18 & .07 & 790 \\
\hline 100 & .40 & .30 & .19 & .05 & 501 \\
\hline \multicolumn{6}{|c|}{$\beta n=1$} \\
\hline 5 & .66 & .19 & .08 & .00 & 742 \\
\hline 10 & .60 & .24 & .11 & .03 & 622 \\
\hline 15 & .47 & .29 & .15 & .07 & 795 \\
\hline 20 & .41 & .30 & .17 & .08 & 620 \\
\hline 25 & .43 & .28 & .18 & .07 & 787 \\
\hline 30 & .41 & .29 & .19 & .08 & 768 \\
\hline 35 & .41 & .29 & .18 & .07 & 840 \\
\hline 40 & .41 & .28 & .19 & .07 & 784 \\
\hline 100 & .39 & .29 & .20 & .05 & 512 \\
\hline
\end{tabular}

Table 6: Fraction of strategic voters supporting policy ranked 1 ... 4. Policy ranked 1 is the winner. Data on policies ranked 5 and 6 are not reported because the supporting fraction is very small.

The fact that individuals are not able to coordinate on a socially better outcome reflects a sort of "race to the bottom" in the voting process: as individuals understand that others might coordinate on voting an obligation that is odious to them, they react by voting another (less odious) obligation.

\section{Conclusions}

In this paper I have developed a simple model of social interaction, where individual actions have (positive or negative) externalities on the well being of others, and individuals are allowed to vote by majority ruling for a ban or a prescription. Assuming no compensations among individuals are contractable 


\begin{tabular}{rccccc}
\hline \multicolumn{5}{c}{ Outcomes (\%) } \\
$N$ & Null & Bans & Prescriptions & Sum & Obs \\
\hline 5 & .41 & .24 & $.35=.2$ & 1 & 832 \\
10 & .34 & .37 & .28 & 1 & 619 \\
15 & .22 & .23 & .54 & 1 & 774 \\
20 & .09 & .11 & .81 & 1 & 570 \\
25 & .04 & .04 & .92 & 1 & 815 \\
30 & .01 & .01 & .98 & 1 & 753 \\
35 & .00 & .00 & 1.00 & 1 & 793 \\
40 & .00 & .00 & 1.00 & 1 & 790 \\
100 & .00 & .00 & 1.00 & 1 & 501 \\
\hline & & & $\beta n=1$ & & \\
5 & .24 & .24 & .53 & 1 & 742 \\
10 & .11 & .34 & .55 & 1 & 622 \\
15 & .01 & .11 & .87 & 1 & 795 \\
20 & .00 & .02 & .97 & 1 & 620 \\
25 & .00 & .00 & 1.00 & 1 & 787 \\
30 & .00 & .00 & 1.00 & 1 & 768 \\
35 & .00 & .00 & 1.00 & 1 & 840 \\
40 & .00 & .00 & 1.00 & 1 & 784 \\
100 & .00 & .00 & 1.00 & 1 & 512 \\
\hline & & & & &
\end{tabular}

Table 7: Strategic outcomes

the social optimum is to place no restrictions on individual behavior if total externalities are low enough, and impose a ban on some specific action if total externalities are higher. Prescriptions are optimal only when a large number of people has a preference over a given action, but still there is a minority that would otherwise choose some other action, and the (negative) externalities from this minority are relevant.

I then turned to private incentives to vote. In pairwise competition policy cycles naturally arise, but if individuals have to choose among all possible bans and prescriptions, the latter always prevail under sincere voting. Except for the case when voting involves only a limited number $(<30)$ of agents this result is also robust to strategic voting.

Thus, the answers to the questions in the title are: yes, we would be better off 
in restricting the number of prescriptions that force individual behavior in favor of more bans, which clearly identify harmful actions; however no, it is unlikely that this could be achieved by majority ruling, given that self-interested voters will tend to support positive obligations. Put differently and more provocatively, a liberal state cannot be directly and liberally enforced. This result stresses the importance of constitutions as a way to provide efficient frameworks for policy discussion. 


\section{A Individual payoffs for different policy options}

In the following, expected payoffs from a cycle of random interactions where all individuals play once are reported, for all different types of individuals and under each policy. Types are indexed as in table 1. Thus, each individual plays once as the active player, and on average $n$ times as a passive player.

I assume that, at any moment in time, all agents are equally likely to act. Hence, the expected payoffs from just one random interaction, where an individual of type $t$ has a probability $1 / N$ of being the active player and a probability $n / N$ of being the passive player is just $\pi_{t}=\Pi_{t} / N$.

\section{A.1 Null policy}

$$
\begin{aligned}
& \Pi_{1}=\alpha+\beta n\left(p_{1}+p_{2}-p_{3}-p_{4}\right) \\
& \Pi_{2}=\alpha+\beta n\left(p_{1}+p_{2}-p_{5}-p_{6}\right) \\
& \Pi_{3}=\alpha+\beta n\left(p_{3}+p_{4}-p_{1}-p_{2}\right) \\
& \Pi_{4}=\alpha+\beta n\left(p_{3}+p_{4}-p_{5}-p_{6}\right) \\
& \Pi_{5}=\alpha+\beta n\left(p_{5}+p_{6}-p_{1}-p_{2}\right) \\
& \Pi_{6}=\alpha+\beta n\left(p_{5}+p_{6}-p_{3}-p_{4}\right)
\end{aligned}
$$

\section{A.2 Prescription to play A}

$$
\begin{aligned}
& \Pi_{1}=\alpha+\beta n \\
& \Pi_{2}=\alpha+\beta n \\
& \Pi_{3}=-\alpha-\beta n \\
& \Pi_{4}=0 \\
& \Pi_{5}=-\alpha-\beta n \\
& \Pi_{6}=0
\end{aligned}
$$




\section{A.3 Prescription to play B}

$$
\begin{aligned}
& \Pi_{1}=-\alpha-\beta n \\
& \Pi_{2}=0 \\
& \Pi_{3}=\alpha+\beta n \\
& \Pi_{4}=\alpha+\beta n \\
& \Pi_{5}=0 \\
& \Pi_{6}=-\alpha-\beta n
\end{aligned}
$$

\section{A.4 Prescription to play $\mathrm{C}$}

$$
\begin{aligned}
& \Pi_{1}=0 \\
& \Pi_{2}=-\alpha-\beta n \\
& \Pi_{3}=0 \\
& \Pi_{4}=-\alpha-\beta n \\
& \Pi_{5}=\alpha+\beta n \\
& \Pi_{6}=\alpha+\beta n
\end{aligned}
$$

\section{A.5 Ban on A}

$$
\begin{aligned}
& \Pi_{1}=0+\beta n\left(-p_{2}-p_{3}-p_{4}\right) \\
& \Pi_{2}=0+\beta n\left(-p_{1}-p_{5}-p_{6}\right) \\
& \Pi_{3}=\alpha+\beta n\left(p_{2}+p_{3}+p_{4}\right) \\
& \Pi_{4}=\alpha+\beta n\left(-p_{1}+p_{2}+p_{3}+p_{4}-p_{5}-p_{6}\right) \\
& \Pi_{5}=\alpha+\beta n\left(p_{1}+p_{5}+p_{6}\right) \\
& \Pi_{6}=\alpha+\beta n\left(p_{1}-p_{2}-p_{3}-p_{4}+p_{5}+p_{6}\right)
\end{aligned}
$$

\section{A.6 Ban on B}

$$
\begin{aligned}
& \Pi_{1}=\alpha+\beta n\left(p_{1}+p_{2}+p_{4}\right) \\
& \Pi_{2}=\alpha+\beta n\left(p_{1}+p_{2}-p_{3}+p_{4}-p_{5}-p_{6}\right) \\
& \Pi_{3}=0+\beta n\left(-p_{1}-p_{2}-p_{4}\right) \\
& \Pi_{4}=0+\beta n\left(-p_{3}-p_{5}-p_{6}\right) \\
& \Pi_{5}=\alpha+\beta n\left(-p_{1}-p_{2}+p_{3}-p_{4}+p_{5}+p_{6}\right) \\
& \Pi_{6}=\alpha+\beta n\left(p_{3}+p_{5}+p_{6}\right)
\end{aligned}
$$


A.7 Ban on $\mathrm{C}$

$$
\begin{aligned}
& \Pi_{1}=\alpha+\beta n\left(p_{1}+p_{2}-p_{3}-p_{4}-p_{5}+p_{6}\right) \\
& \Pi_{2}=\alpha+\beta n\left(p_{1}+p_{2}+p_{6}\right) \\
& \Pi_{3}=\alpha+\beta n\left(-p_{1}-p_{2}+p_{3}+p_{4}+p_{5}-p_{6}\right) \\
& \Pi_{4}=\alpha+\beta n\left(p_{3}+p_{4}+p_{5}\right) \\
& \Pi_{5}=0+\beta n\left(-p_{1}-p_{2}-p_{6}\right) \\
& \Pi_{6}=0+\beta n\left(-p_{3}-p_{4}-p_{5}\right)
\end{aligned}
$$




\section{References}

Kenneth Arrow. A difficulty in the concept of social welfare. Journal of Political Economy, 58(4):328-346, August 1950.

Jeremy Bentham. An introduction to the principles of morals and legislation. Payne, London, 1789.

Isaiah Berlin. Two concepts of liberty. The Clarendon Press, Oxford, 1958.

D. Black. On the rationale of group decision making. Journal of Political Economy, 56:23-34, 1948.

Ronald H. Coase. The problem of social cost. Journal of Law and Economics, $3: 1-44,1960$.

Marquis de Condorcet. Essai sur l'application de l'analyse à la probabilité des décisions rendues à la pluralité des voix. L'Imprimerie Royale, Paris, 1785.

P. Coughlin and S. Nitzan. Electoral outcomes with probabilistic voting and nash social welfare maxima. Journal of Public Economics, 15:113-121, 1981.

O.A. Davis, M. de Groot, and M.J. Hinich. Social preference orderings and majority rule. Econometrica, 40:147-157, 1972.

Maurice Duverger. Party Politics and Pressure Groups. Thomas Y. Crowell, New York, 1972.

Francis T. Edgeworth. Mathematical psychics: An essay on the application of mathematics to the moral sciences. Kegan Paul, London, 1881.

J.S. Gans and M. Smart. Majority voting with single crossing preferences. Journal of Public Economics, 59:219-237, 1996.

Allan Gibbard. Manipulation of voting schemes: a general result. Econometrica, 41(4):587-601, 1973.

J.-M. Grandmont. Intermediate preferences and the majority rule. Econometrica, 46:317-330, 1978

M.J. Hinich. Equilibrium in spatial voting: The median voter result is an artifact. Journal of Economic Theory, 16:208-219, 1977.

M.J. Hinich, O.J. Ledyard, and P.C. Ordershook. Nonvoting and the existence of equilibrium under majority vote. Journal of Economic Theory, 44:144-153, 1972 .

J.O. Ledyard. The paradox of voting and candidate competition: A general equilibrium analysis. In The Handbook of Experimental Economics, pages 777-794. Princeton University Press, Princeton, N.J., 1981. 
O.J. Ledyard. The pure theory of large two-candidate elections. Public Choice, 44:7-41, 1984.

Alfred Marshall. Principles of economics. Macmillan, London, 1890.

David P. Myatt. On the theory of strategic voting. Review of Economic Studies, 74(1):255-281, 2007.

Robert Nozick. Anarchy State and Utopia. Basic Books, New york, 1974.

Torsten Persson and Guido Tabellini. The Economic Effects of Constitutions: What do the Data Say? The MIT Press, Cambridge, MA, 2003.

Arthur C. Pigou. The economics of welfare. Macmillan, London, 1920.

C.R. Plott. A notion of equilibrium and its possibility under majority rule. American Economic Review, 57:787-806, 1967.

Matteo Richiardi. Jesus vs. Hillel. from moral to social norms and back. European Journal European Journal of Economic and Social Systems, forthcoming, 2006.

P. Rothstein. Order restricted preferences and majority rule. Social Choice and Welfare, 7:331-442, 1990.

Mark A. Satterthwaite. Strategy-proofness and arrow's conditions: Existence and correspondence theorems for voting procedures and social welfare functions. Journal of Economic Theory, 10:187-217, 1975.

Amartya Sen. The impossibility of a paretian liberal. Journal of Political Economy, 78(1):152-157, January-February 1970.

Amartya Sen. The possibility of social choice. American Economic Review, 89, July 1999.

Michele Sonnessa. The jas (java agent-based simulation) library. In R. Leombruni and M. Richiardi, editors, Industry and Labor Dynamics: The Agentbased Computational Economics Approach", Singapore, 2004. World Scientific Press.

Leigh Tesfatsion. Agent-based computational economics: A constructive approach to economic theory. In Leigh Tesfatsion and Kenneth L. Judd, editors, Handbook of Computational Economics, volume 2: Agent-Based Computational Economics, chapter 16, pages 831-880. Elsevier, 2006.

Gordon Tullock and Colin D. Campbell. Computer simulation of a small voting system. Economic Journal, 80(317):97-104, 1970.

Friedrich August von Hayek. The Constitution of Liberty. University of Chicago Press, Chicago, 1960.

Oliver E. Williamson. The new institutional economics: Taking stock, looking ahead. Journal of Economic Literature, XXXVIII:595-613, September 2000. 